Internist $2017 \cdot 58: 1264-1271$

https://doi.org/10.1007/s00108-017-0338-5

Online publiziert: 18. Oktober 2017

c) Springer Medizin Verlag GmbH 2017

Redaktion

H. Haller, Hannover

CrossMark

\author{
S. David' • F. M. Brunkhorst ${ }^{2,3,4}$ \\ ${ }^{1}$ Klinik für Nieren- und Hochdruckerkrankungen, Internistische Intensivmedizin, Medizinische Hochschule \\ Hannover, Hannover, Deutschland \\ ${ }^{2}$ Zentrum für Klinische Studien (ZKS), Universitätsklinikum Jena, Jena, Deutschland \\ ${ }^{3}$ Center for Sepsis Control and Care (CSCC), Universitätsklinikum Jena, Jena, Deutschland \\ ${ }^{4}$ Klinik für Anästhesiologie und Intensivmedizin, Universitätsklinikum Jena, Jena, Deutschland
}

\title{
Sepsis-3
}

\section{Was ist gesichert in der Therapie?}

\section{Mit einer Mortalität von knapp $60 \%$ ist der septische Schock auch im 21. Jahrhundert ein tödliches Syndrom, das einer frühen Diagnose sowie einer raschen Einleitung differenzierter Therapien bedarf. Im Jahr 2016 wurde die Sepsisdefinition von 1992 bzw. 2003 abermals erneuert. Sie fokussiert sich nun auf die zentralen Komponenten fehlregulierte Wirtsantwort und Organdysfunktion. Außerdem wurde eine neue internationale Leitlinie publiziert, auf deren Inhalt im vorliegenden Beitrag besonders eingegangen wird.}

\section{Historische Aspekte}

Das Wort Sepsis geht auf Hippocrates zurück und bedeutet aus dem Griechischen übersetzt in etwa so viel wie Fäulnis. Bereits die erste Definition von $\mathrm{Hu}$ go Schottmüller im Jahr 1914 machte deutlich, dass es sich per se nicht um eine Erkrankung, sondern um ein Syndrom mit einer pathologischen Wirtsreaktion auf eine Infektion handelt. Somit ist die Bezeichnung Fäulnis wenig treffend, was nochmal durch die Tatsache deutlich wird, dass Patienten mit Sepsis letztendlich nicht an der Infektion, sondern an der Wirtsantwort sterben.

Auf der Basis dieser Überlegungen wurde die initiale Sepsisdefinition 1989 überarbeitet. Eine Expertenkommission führte ohne echte wissenschaftliche Evidenz das Konzept des Syndroms der systemischen Inflammationsreaktion („systemic inflammatory response syndrome“ [SIRS]) ein [2]. Daraufhin wurde bei nachgewiesenem SIRS im Kontext einer Infektion von Sepsis gesprochen und ein theoretisches Kontinuum der Progression des Syndroms geschaffen [4]:

- Stufe 1: Sepsis

- Stufe 2: schwere Sepsis (mit Organdysfunktion)

- Stufe 3: septischer Schock

Die 4 Kriterien zur Diagnosestellung eines SIRS waren Fieber oder Hypothermie, Tachykardie, Tachypnoe sowie Leukozytose oder Leukopenie, von denen 2 positiv sein mussten. Diese weichen Kriterien haben zu einer relativen Überidentifikation mit konsekutiv hoher Prävalenz des Syndroms - und Optimierung von Krankenhauserlösen - geführt.

\) Das Konzept des SIRS wurde 1989 ohne echte wissenschaftliche Evidenz eingeführt

Um diesen Schwachstellen auf wissenschaftlicher Basis zu begegnen, hat ein internationales Expertenkonsortium 2016 eine neue Definition in drei JAMA-Veröffentlichungen publiziert [12-14]. Anfang 2017 folgte außerdem die Veröffentlichung der neuen internationalen Sepsisleitlinie der Surviving Sepsis Campaign (SSC; $[8,9]$ ).

\section{Sepsis-3}

\section{Konsensusdefinition der Sepsis}

Im Kontext der neuen, dritten Definition ist der Terminus „Sepsis-3“ geschaffen worden. Zum einen handelt es sich um die dritte Konsensuskonferenz, zum anderen wurden in einer sehr aufwendigen Analyse von 850.000 Patienten mit einer Infektion 3 einfache klinische Indikatoren für das Screening der Organdysfunktion identifiziert (sogenannter Quick Sequential Organ Failure Assessment [qSOFA] Score, s. unten). Zu guter Letzt wurden die Ergebnisse und Entscheidungsprozesse in 3 konsekutiven JAMA-Beiträgen veröffentlicht [12-14]. Die dritte internationale Konsensusdefinition lautet:

Sepsis wird definiert als lebensbedrohliche Organdysfunktion, die durch eine fehlregulierte Wirtsantwort auf eine Infektion hervorgerufen wird.

Erwähnenswert für die Einordnung dieser neuen Definition ist die Tatsache, dass die Diagnose der zugrunde liegenden Infektion nicht Gegenstand von Sepsis-3 war und ausschließlich über die Empfehlungen der verantwortlichen Fachgesellschaften definiert wird. Nach der Publikation von Sepsis-3 wurde der vermeintliche Wegfall des SIRS teilweise sehr emotional diskutiert. Die Tatsache, dass die SIRS-Kriterien nach wie vor eine wichtige Rolle bei der Diagnosestellung der Erkrankung spielen, nicht aber Gegenstand der Definition an sich sind, wurde von den Kollegen offensichtlich übersehen. Das Konzept eines Syndroms der systemischen Inflammation ist keinesfalls einfach verschwunden.

\section{Organdysfunktion}

Die Publikation von Mervin Singer et al. gibt darüber hinaus eine interessante Empfehlung, wie wir als Kliniker 
Tab. 1 Sequential Organ Failure Assessment (SOFA) Score

\section{Score}

\begin{tabular}{|c|c|c|c|c|c|}
\hline Organsystem & 0 & 1 & 2 & 3 & 4 \\
\hline \multicolumn{6}{|l|}{ Lunge } \\
\hline $\begin{array}{l}\mathrm{p}_{\mathrm{a}} \mathrm{O}_{2} / \mathrm{F}_{1} \mathrm{O}_{2} \\
(\mathrm{~mm} \mathrm{Hg})\end{array}$ & $>400$ & $<400$ & $<300$ & $\begin{array}{l}<200+\text { Beat- } \\
\text { mung }\end{array}$ & $<100+$ Beatmung \\
\hline \multicolumn{6}{|l|}{ Koagulation } \\
\hline $\begin{array}{l}\text { Thrombozyten } \\
\left(\cdot 10^{3} / \mu \mathrm{l}\right)\end{array}$ & $\geq 150$ & $<150$ & $<100$ & $<50$ & $<20$ \\
\hline \multicolumn{6}{|l|}{ Leber } \\
\hline Bilirubin ( $\mu \mathrm{mol} / \mathrm{l})$ & $<20$ & $20-32$ & $33-101$ & $102-204$ & $>204$ \\
\hline \multicolumn{6}{|l|}{ Kardiovaskulär } \\
\hline MAD (mm Hg) & $\geq 70$ & $<70$ & - & - & - \\
\hline $\begin{array}{l}\text { Vasopressor } \\
(\mu \mathrm{g} / \mathrm{kg} / \mathrm{min})\end{array}$ & - & - & $\begin{array}{l}\text { Dopamin }<5 \\
\text { oder Dobut- } \\
\text { amin (dosisun- } \\
\text { abhängig) }\end{array}$ & $\begin{array}{l}\text { Dopamin } 5,1-15 \\
\text { oder Epine- } \\
\text { phrin } \leq 0,1 \text { oder } \\
\text { Norepine- } \\
\text { phrin } \leq 0,1\end{array}$ & $\begin{array}{l}\text { Dopamin }>15 \\
\text { Epinephrin }>0,1 \\
\text { Norepinephrin }>0,1\end{array}$ \\
\hline \multicolumn{6}{|c|}{ Zentrales Nervensystem } \\
\hline GCS-Score & 15 & $13-14$ & $10-12$ & $6-9$ & $<6$ \\
\hline \multicolumn{6}{|l|}{ Niere } \\
\hline Kreatinin $(\mu \mathrm{mol} / \mathrm{l})$ & $<110$ & $110-170$ & $171-299$ & $300-440$ & $>440$ \\
\hline $\begin{array}{l}\text { Urinausscheidung } \\
\text { (ml/Tag) }\end{array}$ & - & - & - & $<500$ & $<200$ \\
\hline $\begin{array}{l}\mathrm{F}_{1} \mathrm{O}_{2} \text { Fraktion des eir } \\
\text { Druck; } p_{a} \mathrm{O}_{2} \text { arteriell }\end{array}$ & $\begin{array}{l}\text { ingeatme } \\
\text { ler Sauer }\end{array}$ & $\begin{array}{l}\text { n Sauerstc } \\
\text { fffpartialdr }\end{array}$ & $\begin{array}{l}\text { iffs; GCS Glasgow } \\
\text { uck }\end{array}$ & Coma Scale; MAD n & ittlerer arterieller \\
\hline
\end{tabular}

die „Organdysfunktion“ schnell objektiv erkennen sollen. Hierzu wird eine Veränderung des - zugegebenermaßen komplexen - Sequential Organ Failure Assessment (SOFA) Score um $\geq 2$ Punkte vorgeschlagen (•Tab. 1). Da der Score, der 6 Organsysteme nach 4 Schweregraden der Dysfunktion einstuft und damit 0-24 Punkte umfasst, in der Routinepraxis zum bettseitigen Screening ungeeignet ist, haben die Autoren anhand einer retrospektiven Analyse von 850.000 Patientenakten an 177 internationalen Kliniken die drei wesentlichen Prädiktoren einer Organdysfunktion wissenschaftlich herausgearbeitet und diese als „Quick SOFA“ (qSOFA) bezeichnet. qSOFA ist als positiv zu werten, wenn mindestens 2 von 3 positiven Befunden folgender Stratifizierung vorliegen:

- Atemfrequenz $\geq 22 / \mathrm{min}$

- Verändertes Bewusstsein (Glasgow

Coma Scale [GCS] <15)

- Systolischer Blutdruck $\leq 100$ mm Hg

Wichtig ist hierbei, dass qSOFA ausschließlich als Screeninginstrument zur raschen Identifikation von Sepsisrisiko- patienten eingesetzt werden soll. Dieser Score ist validiert für den präklinischen Gebrauch, die Notaufnahme und die Normalstation, nicht aber für die Intensivstation. Zur tatsächlichen Diagnosestellung muss entsprechend einem vorgeschlagenen Algorithmus bei den qSOFA-positiven Patienten letztendlich doch eine einmalige Bestimmung des SOFA-Scores erfolgen (- Tab. 1). In der Publikation wurde ein Algorithmus zur bettseitigen Vorgehensweise vorgeschlagen (• Abb. 1).

\section{》) qSOFA dient als Scree- ninginstrument zur raschen Identifikation von Sepsisrisikopa- tienten}

Da die Organdysfunktion nun fundamentaler Bestandteil der Definition ist, liegt auf der Hand, dass der alte Begriff der schweren Sepsis entfällt. Er wurde als Sepsis plus Organdysfunktion verstanden. Man spricht also nur noch von „Sepsis“ und „septischem Schock“, der
Tab. 2 Vergleich der alten und neuen Gra-

duierungssysteme der Sepsisleitlinien 2012

und 2016

\begin{tabular}{|l|l|l|}
\hline & $\mathbf{2 0 1 6}$ & $\mathbf{2 0 1 2}$ \\
\hline Stärke & Stark & 1 \\
\hline & Schwach & 2 \\
\hline Qualität & Hoch & A \\
& Moderat & B \\
\hline & Niedrig & C \\
\hline $\begin{array}{l}\text { Starke Empfehlung } \\
\text { ohne Evidenz }\end{array}$ & BPS & D \\
\hline $\begin{array}{l}\text { rig } \\
\text { BPS „Best practice statements }\end{array}$ & - \\
\hline
\end{tabular}

ebenfalls neu definiert wurde und nun als zusätzliches obligates Kriterium eine Veränderung des Serumlaktats als Marker der gestörten Mikrozirkulation beinhaltet.

\section{Septischer Schock}

Der septische Schock ist eine Untergruppe der Sepsis, bei der die vorliegenden zirkulatorischen zellulären und metabolischen Störungen so ausgeprägt sind, dass die Sterblichkeit substanziell zunimmt. Konkret handelt es sich um eine Sepsis mit folgenden zusätzlichen Komponenten:

- Katecholaminpflichtigkeit zur Aufrechterhaltung eines mittleren arteriellen Drucks (MAD) $\geq 65 \mathrm{~mm} \mathrm{Hg}$

- Serumlaktat >2 mmol/l (>18 mg/dl) trotz adäquater Volumensubstitution

\section{Öffentlichkeitsarbeit und Laiendefinition}

$\mathrm{Zu}$ guter Letzt hat sich die Konsensuskonferenz mit dem wichtigen Thema der Öffentlichkeitsarbeit befasst. Jeder Kliniker kennt das Problem der mangelnden Aufklärung vor allem der deutschen Bevölkerung und hat den verwirrenden Begriff der „Blutvergiftung“ im Patienten- bzw. Angehörigengespräch schon oft zu hören bekommen. Manch Angehöriger erinnert sich bei der Konfrontation mit einer Sepsisdiagnose vor allem an die Worte der geschätzten Großmutter, die den Enkelkindern lebhaft von „einem aufsteigenden roten Strich am Arm, der bei Erreichen des Herzens unweigerlich 
zum sofortigen Tode führen sollte", erzählte. Um die Aufmerksamkeit der Bevölkerung und das Verständnis zu verbessern, wurde eine Laiendefinition der Sepsis vorgeschlagen. Nach unserer eigenen Einschätzung ist diese im täglichen Gebrauch nützlich und hilft, das komplexe Erkrankungsbild einfach zu erklären:

Sepsis ist eine lebensbedrohliche Erkrankung, bei der die Reaktion des Körpers auf eine Infektion zu einer Schädigung der eigenen Gewebe und Organe führt.

Anfang des Jahres hat die SSC die neue internationale Sepsisleitlinie in den Fachzeitschriften Critical Care Medicine [9] und Intensive Care Medicine [8] publiziert. Dies geschieht alternierend mit den Empfehlungen der Deutschen Sepsis-Gesellschaft (DSG) etwa alle 4 Jahre. Im Folgenden haben wir die Neuerungen mit Fokussierung auf Therapieaspekte zusammengefasst.

\section{Internationale Leitlinie 2016}

Eine wichtige Änderung der aktuellen Leitlinie liegt im Graduierungssystem der Empfehlungen (• Tab. 2) - auch wenn die Sinnhaftigkeit einer erneuten Änderung der Graduierung fraglich bleibt, nachdem man sich an ein mittlerweile flächendeckend eingesetztes System gewöhnt hat. Außerdem werden Behandlungsbereiche, für oder gegen die es keine ausreichende Evidenz gibt, neuerdings konkret benannt und verbleiben ohne Empfehlung. Bei klinisch routinemäßig implementierten Therapiestrategien mit guter theoretischer Grundlage und stimmiger Experteneinschätzung, aber mit mangelnder Studienlage wurde zudem der Begriff der "best practice statements“ eingeführt. Im Folgenden fassen wir die wesentlichen Aspekte mit subjektivem Kommentar zusammen.

\section{Screening}

Analog zu den Erfahrungen im Staat New York (Stichwort Rory's Regulations) wird auch in der internationalen Leitlinie ein System für die Implementierung eines Sepsisprogramms zur Verbesserung der Behandlung empfohlen. Dass Krankenhäuser diese Programme und ihre Rea-

Internist 2017·58:1264-1271 https://doi.org/10.1007/s00108-017-0338-5

(c) Springer Medizin Verlag GmbH 2017

\section{S. David · F. M. Brunkhorst}

\section{Sepsis-3. Was ist gesichert in der Therapie?}

\section{Zusammenfassung}

Hugo Schottmüller erkannte bereits vor über 100 Jahren, dass es sich bei der Sepsis um ein Syndrom handelt, bei dem nicht die Infektion an sich, sondern eine Überreaktion des Organismus auf eine periodische Einschwemmung von Krankheitserregern unsere Patienten schädigt. Vor etwa 25 Jahren wurde entsprechend diesem Konzept der Begriff des Syndroms der systemischen Inflammationsreaktion (,systemic inflammatory response syndrome" [SIRS]) geprägt. Er wird im klinischen Alltag quasi ständig gebraucht. Im Jahr 2016 hat sich eine Expertenkommission bemüht, eine dritte Sepsisdefinition (Sepsis-3) zu erstellen, die auf wissenschaftlichen Daten beruhen sollte. In den Vordergrund rückt nun die fehlregulierte Wirtsantwort auf eine Infektion zusammen mit der Organdysfunktion als obligate Voraussetzung der Diagnosestellung. In diesem Beitrag wird die neue Definition im Detail erläutert. Zudem wird die Anfang des Jahres 2017 erschienene internationale Leitlinie zusammengefasst und kommentiert.

Schlüsselwörter

Septischer Schock · Syndrom der systemischen Inflammationsreaktion - Leitlinie · Scores des Organversagens · Flüssigkeitstherapie

\section{Sepsis-3. What has been confirmed in therapy?}

\section{Abstract}

Hugo Schottmüller realized already over 100 years ago that sepsis is a syndrome that occurs as a consequence of an infection defined by a systemic overreaction to a periodic invasion of bacilli. Consistent with this old concept, the term "systemic inflammatory response syndrome" (SIRS) was coined about 25 years ago and has been regularly used ever since. In 2016, a sepsis task force was formed to re-evaluate the current definition on a scientific basis. The task force suggested a third definition (sepsis-3) of the disease that now focuses both on the pathological host response and on organ dysfunction as obligatory key features to diagnose sepsis. In this review article, the definition is explained in detail and we summarize the novel international sepsis guideline from 2017 with personal commentaries.

Keywords

Septic shock - Systemic inflammatory response syndrome · Guideline · Organ dysfunction scores · Fluid therapy lisierung offenlegen müssen, ist jedoch bisher kein Thema. In diesem Kontext soll kurz der Hintergrund zu Rory's Regulations erläutert werden. Rory war ein 12-jähriger Junge, der im April 2012 nach einer Bagatellverletzung beim Schulsport an einer unerkannten Sepsis mit „toxic shock syndrome“ verstarb. Die immense Öffentlichkeitsarbeit der Eltern hat führende Politiker des Staates New Yorknicht nur dazu veranlasst, gesetzliche Rahmenbedingungen zu schaffen, die die Krankenhäuser verpflichten, evidenzbasierte Protokolle zur Sepsisdiagnose und -therapie flächendeckend umzusetzen. Die Kliniken müssen dem Staat darüber auch dezidiert Bericht erstatten. Erste Ergebnisse von etwa 50.0000 septischen Pati- enten aus 149 New Yorker Kliniken sind im Juni 2017 veröffentlicht worden [11].

\section{Diagnostik}

Bei Verdacht auf Sepsis sollten so schnell als möglich - unbedingt jedoch innerhalb der ersten $3 \mathrm{~h}$ - Blutkulturen abgenommen werden. Vorausgesetzt, dass keine zeitliche Verzögerung entsteht, soll dies noch vor Verabreichung eines Breitspektrumantibiotikums erfolgen.

\section{Fokussuche}

Bei unklarem, aber potenziell sanierbarem Fokus wird eine schnellstmögliche anatomische Diagnostik, also eine Bildgebung, empfohlen. Falls intravaskuläre 


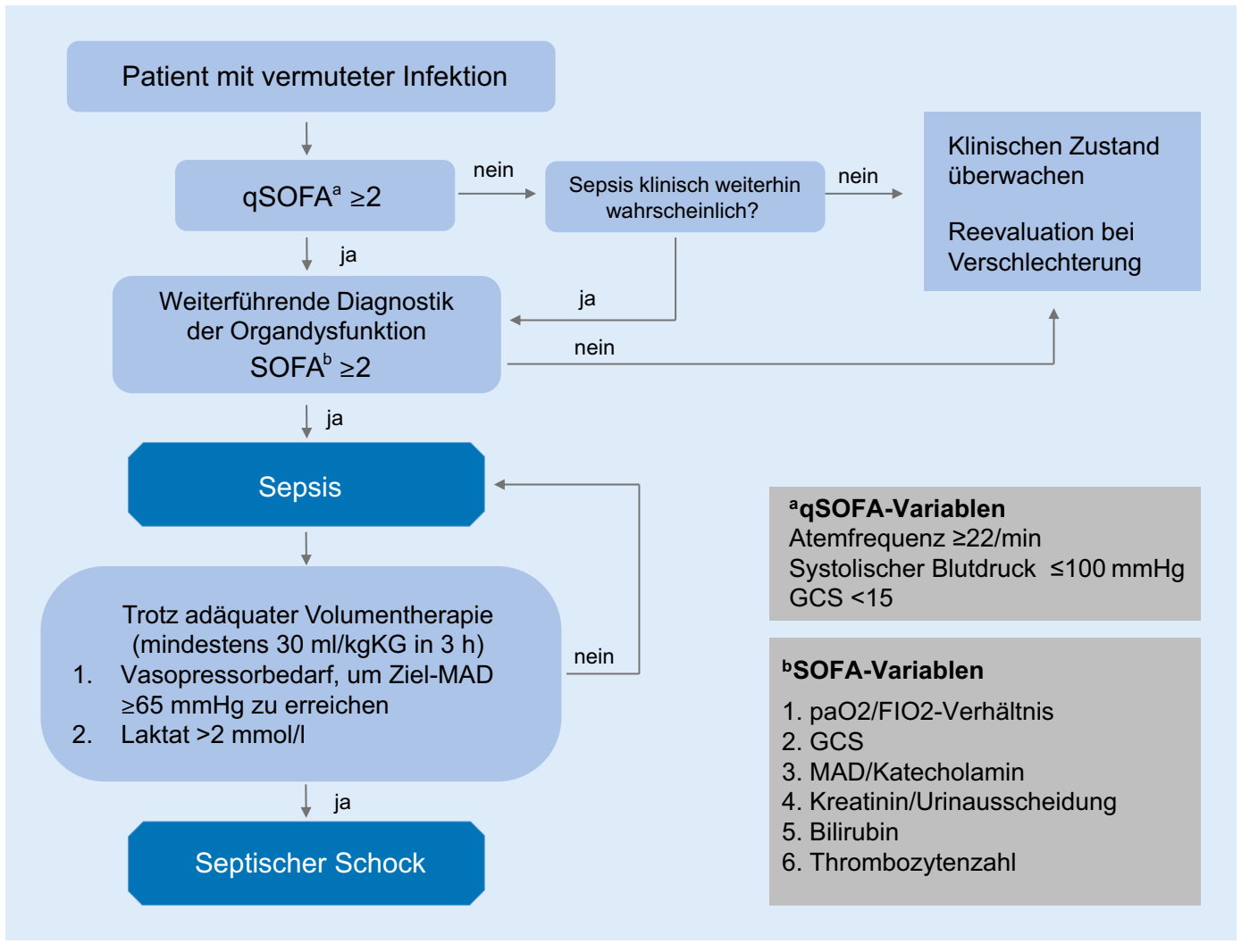

Abb. $1<$ Vorschlag eines Algorithmus zur Sepsisdiagnostik. $\mathrm{F}_{1} \mathrm{O}_{2}$ Fraktion des eingeatmeten Sauerstoffs; GCS Glasgow Coma Scale; $M A D$ mittlerer arterieller Druck; $p_{a} \mathrm{O}_{2}$ arterieller Sauerstoffpartialdruck; $q S O$ $F A_{\text {"Q }}$ Quick SOFA"; SOFA Sequential Organ Failure Assessment. (Adaptiert nach [14])

Zugänge einen möglichen Fokus darstellen, sollten diese unverzüglich entfernt werden. Hierbei dürfen vor allem permanente Katheter wie Ports oder getunnelte Vorhofdialysekatheter nicht vergessen werden. Sie müssen gegebenenfalls notfallmäßig operativ entfernt werden.

\section{Antimikrobielle Therapie}

Innerhalb der ersten Stunde soll eine empirische parenterale Antiinfektivatherapie mit einem oder mehreren Präparaten erfolgen. Dabei sind das mutmaßliche Erregerspektrum (Pilze, Viren, „multidrug resistance“) und die Gewebepenetranz je nach klinischem Fokus zu berücksichtigen.

\section{) Eine Deeskalation der antimikrobiellen Therapie ist so schnell wie möglich durchzuführen}

Nur bei septischem Schock wird eine, wenngleich schwache, Empfehlung für eine Kombinationstherapie ausgespro- chen. Dies gilt explizit nicht für die einfache Bakteriämie oder Sepsis; interessanterweise auch nicht für die neutropene Sepsis.

Die empirische Therapie soll nach Erregeridentifikation und Testung der minimalen Hemmkonzentration (MHK) entsprechend den üblichen TarragonaPrinzipien (•Infobox 1) erfolgen. Sowohl bei mikrobiologischem Erregernachweis als auch bei empirischer Kombinationstherapie im septischen Schock ist - nach klinischem Zustand - eine Deeskalation so schnell wie möglich durchzuführen.

Prinzipiell wird eine Therapie über 7-10 Tage für ausreichend erachtet, allerdings gibt es wichtige Ausnahmesituationen:

- Verzögertes klinisches Ansprechen

- Nicht drainierbare Infektion

- Staphylococcus-aureus-Bakteriämie

- Immundefizienz

- Spezielle Virus- oder Pilzinfektionen

Im Gegensatz hierzu soll die Therapiedauer verkürzt werden, wenn ein sehr gutes klinisches Ansprechen beobachtet werden kann. Eine Evaluation, ob eine
Deeskalation möglich ist, sollte täglich erfolgen.

Prokalzitonin (PCT) wird als adäquates Hilfsmittel zur Steuerung der Antibiotikatherapie erwähnt. Dieses Statement bezieht sich zum einen auf die Beendigung einer Therapie nach erfolgreicher Infektkontrolle und zum anderen auf das Absetzen einer initial empirischen Therapie bei Verdacht auf Sepsis mit jedoch nachfolgend fehlender Evidenz, also bei einer irrtümlichen Therapie.

\section{Initiale Flüssigkeitstherapie}

Die Gabe von Kristalloiden ist die initiale Flüssigkeitstherapie der Wahl. Da es sich um eine Notfallsituation handelt, versteht es sich, dass unverzüglich mit der Therapie begonnen werden soll. Bei klinischen Zeichen der Hypoperfusion (Hypotonie oder Laktat $>4 \mathrm{mmol} / \mathrm{l}$ ) sollen innerhalb von $3 \mathrm{~h} 30 \mathrm{ml} / \mathrm{kg} \mathrm{KG}$ balancierte Kristalloide appliziert werden. Ein Patient mit Gewicht von $80 \mathrm{~kg}$ benötigt somit 2,4l in $3 \mathrm{~h}$.

Eine Steuerung der weiteren Flüssigkeitsgaben erfolgt anhand des hämodynamischen Zustands, der regelmäßig re- 


\section{Infobox 1 Fünf Prinzipien der} Tarragona-Strategie

1. Individuelle Risikofaktoren des Patienten ("look at your patient")

2. Lokale Epidemiologie/Resistenzlage („look at your hospital")

3. Breite und schnelle Initialtherapie (,hit hard and early")

4. Welcher mutmaßliche Fokus ("get to the point")?

5. Reevaluation innerhalb von 48 bis $72 \mathrm{~h}$ („focus, focus, focus")

evaluiert werden soll. Dies beinhaltet bei Patienten mit septischem Schock folgende Parameter:

- Herzfrequenz

- MAD

- Blutgasanalyse mit $\mathrm{S}_{\mathrm{a}} \mathrm{O}_{2}$ und Laktat

- Atemfrequenz

- Temperatur

- Urinproduktion

Ein erweitertes hämodynamisches Monitoring wird gefordert, wenn klinisch keine klare Ätiologie des Schocks feststellbar ist. Hier wird besonders auf den hohen Stellenwert dynamischer Vorlastparameter anstelle von statischen Parametern wie dem zentralen Venendruck (ZVD) für die Flüssigkeitsreagibilität hingewiesen. Der empfohlene ZielMAD liegt weiterhin bei $\geq 65 \mathrm{~mm} \mathrm{Hg}$ (auf interindividuelle Differenzen der Hausblutdruckwerte wird in der Leitlinie nicht eingegangen).

Es wird eine schwache - jedoch nach Meinung der Autoren sehr sinnvolle Empfehlung zur Steuerung der Therapie mit dem Ziel einer Laktatnormalisierung ausgesprochen.

\section{Weiteres Flüssigkeitsmanagement}

Solange eine weitere Applikation von kristalloider Flüssigkeit die Hämodynamik verbessert (Stichwort „fluid challenge"), sollte sie weitergeführt werden. Die Volumenreagibilität kann klinisch besonders zuverlässig unter kontinuierlichem Monitoring des Herzzeitvolumens mit einer Autotransfusion, dem sogenannten „passive leg raising test", überprüft werden [3].
Eine schwache Empfehlung mit niedriger Evidenz gibt es für den Einsatz von Albumin bei Patienten, die erhebliche Mengen an Kristalloiden benötigen. Diese schwache Empfehlung mit geringer Evidenzqualität basiert vor allem auf Subgruppen- und Metaanalysen und sollte unseres Erachtens nicht zum unreflektierten Einsatz dieser teuren Substanz führen. Kontrollierte, randomisierte Daten liegen aktuell nicht vor, eine nationale multizentrische Studie ist aber aktuell in Deutschland geplant. Die Leitlinie bleibt dem Leser schuldig, ab wann konkret von erheblichen Flüssigkeitsmengen gesprochen wird. Ausdrücklich nicht empfohlen ist die Verwendung von Hydroxyethylstärke, diese führt zu einer erhöhten Inzidenz der akuten Nierenschädigung und zur Übersterblichkeit [6].

\section{Vasopressoren}

Basierend auf der Pathophysiologie des septischen Schocks mit massivem peripherem Widerstandsverlust ist Norepinephrin nach wie vor das Medikament der ersten Wahl. Bei Nichterreichen des Ziel-MAD $\geq 65 \mathrm{~mm} \mathrm{Hg}$ oder zum Einsparen sehr hoher Norepinephrindosen kann eine zusätzliche Gabe von Vasopressin (bis zu 0,03 U/min) erfolgen. Zum kontinuierlichen Monitoring sollte bei allen Patienten eine invasive Blutdruckmessung erfolgen.

Der Einsatz von Dopamin wird nur bei einer selektierten Subgruppe von Patienten empfohlen; sicher soll kein Einsatz in sogenannter niedriger "Nierendosis“ erfolgen. In den Kliniken der Autoren kommt Dopamin in dieser Indikation de facto jedoch nie zum Einsatz. Dobutamin hingegen wird bei Patienten mit persistierender Hypoperfusion trotz adäquater Flüssigkeits- und Vasopressortherapie empfohlen.

\section{Blutprodukte}

Bei kardial gesunden Patienten sollten Erythrozytenkonzentrate erst ab einem Hämoglobinspiegel <7,0 g/dl transfundiert werden. Frischplasma (FFP) soll nicht zur Korrektur von Gerinnungsparametern oder vor geplanten invasiven Tätigkeiten verabreicht werden.
Auch für die Volumentherapie ist FFP nicht empfohlen. Bei nicht blutenden Patienten sollten Thrombozyten prophylaktisch erst ab einer Reduktion auf $<10.000 / \mathrm{mm}^{3}$ verabreicht werden; bei hohem Blutungsrisiko wird eine Grenze von $20.000 / \mathrm{mm}^{3}$ vorgeschlagen.

\section{Beatmung bei sepsisinduziertem „acute respiratory distress syndrome"}

Das anzustrebende Atemzugvolumen entspricht den üblichen Empfehlungen zur lungenprotektiven Beatmung $\left(\mathrm{V}_{\mathrm{t}}\right.$ : $6 \mathrm{ml} / \mathrm{kg}$ vorhergesagtes Körpergewicht) im Rahmen des Konzepts der permissiven Hyperkapnie. Empfohlen ist ein hoher positiver endexspiratorischer Druck (PEEP) bei jedoch gleichzeitiger Einhaltung eines maximalen Plateaudrucks $\leq 30 \mathrm{~cm} \mathrm{H}_{2} \mathrm{O}$.

Eine starke Empfehlung wird auch für die, unseres Erachtens sehr effiziente, Bauchlagerung bei Patienten mit einem Oxygenierungsindex $\left(\mathrm{p}_{\mathrm{a}} \mathrm{O}_{2} / \mathrm{F}_{\mathrm{I}} \mathrm{O}_{2}\right) \quad \leq 150 \mathrm{~mm} \mathrm{Hg}$ ausgesprochen. Besteht eine derartige Oxygenierungsstörung ( $<150 \mathrm{~mm} \mathrm{Hg}$ ) für weniger als 48 h, kann zusätzlich der Einsatz von Muskelrelaxanzien erwogen werden. Bei Patienten ohne Zeichen der Hypoperfusion sollte ein konservatives Flüssigkeitsregime einem liberalen vorgezogen werden. Trotz relativ schwacher Evidenz wurde eine starke Empfehlung für die Oberkörperhochlagerung (30-45) ausgesprochen.

Für den weiteren Verlauf werden standardisierte Weaning-Protokolle sowie der regelmäßige Einsatz von Spontanatemversuchen empfohlen.

\section{Nierenersatztherapie}

Prinzipiell sind intermittierende Dialyseverfahren den kontinuierlichen Verfahren gleichwertig. Dennoch wird in der Leitlinie ein kontinuierliches Verfahren aufgrund der möglicherweise besseren Steuerung des Flüssigkeitshaushalts bei hämodynamisch instabilen Patienten präferiert, was auch der klinischen Praxis in den Kliniken der Autoren entspricht, aber nie in Studien belegt wurde. Ein erhöhtes Kreatinin oder eine verminder- 

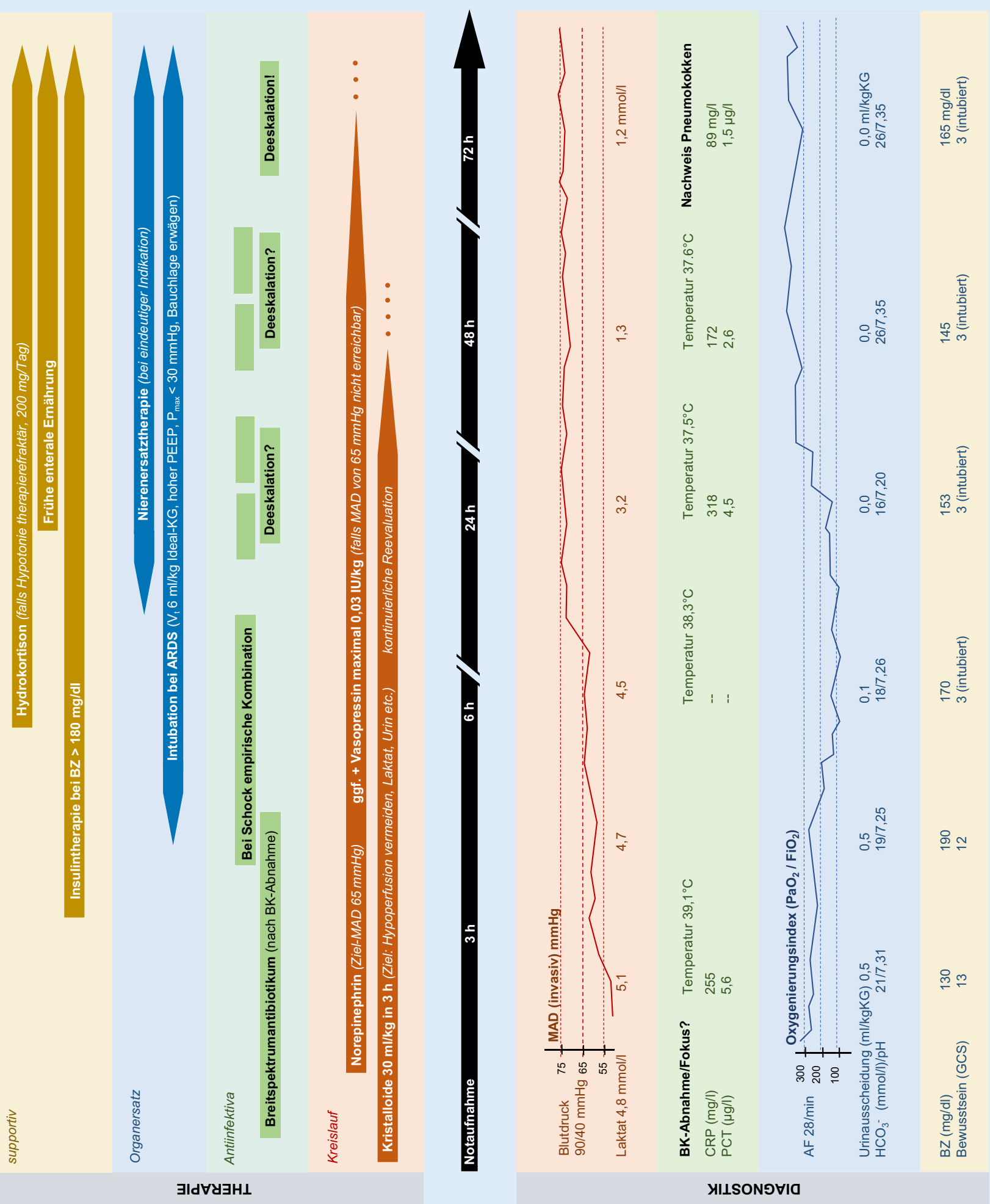

Abb. 2 A Diagnostik und Therapieoptionen der Sepsis im zeitlichen Verlauf. Schematische Darstellung eines Beispielpatienten. AF Atemfrequenz; $A R D S_{\text {" }}$ acute respiratory distress syndrome"; BK Blutkultur; BZ Blutzucker; CRP C-reaktives Protein; GCS Glasgow Coma Scale; MAD mittlerer arterieller Druck; PCT Prokalzitonin; PEEP positiver endexspiratorischer Druck 
te Urinproduktion allein sollte nicht zur Indikationsstellung der Nierenersatztherapie führen.

\section{Blutzuckerkontrolle}

Empfohlen ist eine protokollbasierte Vorgehensweise zur Blutzuckereinstellung mit einem oberen Blutzuckerziel von $\leq 180 \mathrm{mg} / \mathrm{dl}$ (nicht $110 \mathrm{mg} / \mathrm{dl}$ !). Bei 2 aufeinanderfolgenden Werten $>180 \mathrm{mg} / \mathrm{dl}$ wird mit der Insulintherapie begonnen. Bis zum Erreichen eines stabilen Niveaus sind 1- bis 2-stündliche Messungen angeraten, danach alle $4 \mathrm{~h}$. Zusammenfassend überwiegt das Risiko einer Hypoglykämie und der damit verbundenen Komplikationen den potenziellen Nutzen einer strengeren Blutzuckereinstellung.

\section{Ernährung}

Bei Patienten, die enteral ernährt werden können, ist immer eine frühe enterale Ernährung anzustreben; eine frühe parenterale Ernährung ist hingegen nicht empfohlen. Falls keine frühe enterale Ernährung möglich ist, sollen initial Glukoselösungen eingesetzt werden. Ein routinemäßiges Monitoring des gastralen Refluxes ist nicht erforderlich, kann aber bei Ernährungsintoleranz oder hohem Aspirationsrisiko sinnvoll sein (gilt jedoch nicht für chirurgische Patienten). In diesem Fall sollten prokinetische Medikamente und gegebenenfalls postpylorische Ernährungssonden eingesetzt werden. Unter Immunonutrition bekannte Zusatzstoffe wie Selen, Glutamin, Arginin und Carnitin sollen nicht in der Sepsis eingesetzt werden.

\section{Prophylaxe}

Eine Empfehlung zur täglichen Thromboseprophylaxe mit einer Präferenz für niedermolekulare Heparine gegenüber unfraktioniertem Heparin wurde ausgesprochen. Zudem ist eine Kombination mit einer mechanischen Prophylaxe, wann immer möglich, empfohlen. Diese soll auch bei Patienten mit Kontraindikationen einer pharmakologischen Prophylaxe konsequent eingesetzt werden.
Die Stressulkusprophylaxe ist ausdrücklich nur bei Patienten mit erhöhtem Risiko einer gastrointestinalen Blutung empfohlen. Bei der Durchführung sind $\mathrm{H}_{2}$-Blocker den Protonenpumpeninhibitoren gleichwertig.

\section{Adjuvante Therapiestrategien}

Der Einsatz von Hydrokortison sollte - wie bereits in der letzten Auflage der Leitlinie - auf Patienten mit flüssigkeits- und katecholaminrefraktärem Schock begrenzt bleiben (200 mg/Tag kontinuierlich i.v.).

Intravenöse Immunglobuline bei erwachsenen Patienten mit septischem Schock sind bisher nicht empfohlen. Aufgrund fehlender Daten wird außerdem keine Empfehlung für extrakorporale Adsorptionsverfahren sowie den Einsatz von Thrombomodulin ausgesprochen.

Bei Patienten mit hypoperfusionsinduzierter Laktatazidämie und einem $\mathrm{pH} \geq 7,15$ sollte kein Natriumbicarbonat zur Verbesserung der Hämodynamik eingesetzt werden.

In Abb. 2 sind die wesentlichen Empfehlungen sowohl diagnostischer als auch therapeutischer Natur an einem fiktiven Patientenbeispiel im zeitlichen Verlauf grafisch zusammengefasst.

\section{Anmerkungen und Status quo}

Die aktuelle Leitlinie fasst die wirklich gesicherten - also evidenzbasierten - Strategien zusammen. Allerdings kann man festhalten, dass der Hang zu Altbewährtem - Nichtevidenzbasiertem - an einigen Stellen nicht zu übersehen ist.

Die Evidenz aus intensivmedizinischen Studien, dass der ZVD als statischer Vorlastparameter ungeeignet zur Abschätzung des Volumenstatus ist und in dieser Indikation nicht mehr angewendet werden sollte, ist eindeutig. Außerdem haben die transatlantischen Studien ProCESS [7], ARISE [1] und ProMISe [5] zur zielgerichteten Therapie der Sepsis („early goal-directed therapy“) ergeben, dass die $\mathrm{ZVD}$ - und $\mathrm{S}_{\mathrm{cv}} \mathrm{O}_{2}$-basierten Protokolle keinen Unterschied in Bezug auf das Überleben hatten. Auch wenn diese zur ursprünglichen Rivers-Studie von 2001 [10] gegenteiligen Ergebnisse teilweise auf die vor Studieneinschluss revolutionierte Standardtherapie mit schneller Flüssigkeitsgabe bereits in den Notaufnahmen und Normalstationen zurückzuführen sind, unterstreichen sie die generelle Fragwürdigkeit dieser Zielparameter. Dennoch kann man in der Leitlinie lesen, dass zur erweiterten Flüssigkeitstherapie - zumindest nach Beendigung des $3 \mathrm{~h}$-Bundles - ZVD und $\mathrm{S}_{\mathrm{cv}} \mathrm{O}_{2}$ hilfreich sein können. Diese verwirrenden Statements basieren wohl eher auf historischen Umständen als auf evidenzbasierten Fakten.

Im Prinzip sind alle vorgestellten Behandlungskonzepte mit Ausnahme von Antiinfektiva und Fokussanierung nicht kausal gegen das pathophysiologische Problem der Sepsis - nämlich die fehlreguliere Wirtsreaktion - gerichtet, sondern vielmehr supportiver Natur. Die Zusammenarbeit zwischen Klinikern und translationalen Grundlagenforschern ist in diesem Kontext immens wichtig. Eine Verbesserung der Infrastruktur zur Förderung innovativer Bench-to-bedside-Projekte ist von fundamentaler Bedeutung, wenn unsere bis dato ernüchternden therapeutischen Strategien vorangebracht werden sollen.

\section{Fazit für die Praxis}

- Die Mortalität der schwersten Form der Sepsis, des septischen Schocks, beträgt in Deutschland immer noch knapp $60 \%$.

- Seit 2016 gibt es eine neue Sepsisdefinition, in der die Komponenten Organdysfunktion und fehlregulierte Wirtsreaktion in den Vordergrund gerückt wurden.

- Um die öffentliche Sichtbarkeit des Syndroms zu erhöhen und die Patienten- bzw. Angehörigenkommunikation zu verbessern, wurde zudem eine Laiendefinition vorgeschlagen.

- qSOFA wurde als Screeninginstrument der Organdysfunktion zur Identifikation von Risikopatienten auf der Normalstation definiert. Es beinhaltet erstmals wissenschaftlich evaluierte Zielparameter: verändertes Bewusstsein, Atemfrequenz $>22 /$ min und systolischer Blutdruck 
$<100 \mathrm{~mm} \mathrm{Hg}$ (positiv bei $\geq 2$ von 3 Parametern).

- Anfang des Jahres wurde eine neue internationale Sepsisleitlinie mit evidenzbasierten Handlungsempfehlungen veröffentlicht.

- Neue spezifische Therapiestrategien, die die pathologische Wirtsantwort modulieren, gibt es nicht. Dies unterstreicht die Wichtigkeit translationaler Grundlagenforschung.

\section{Korrespondenzadresse}

\section{PD Dr. S. David}

Klinik für Nieren- und Hochdruckerkrankungen, Internistische Intensivmedizin, Medizinische Hochschule Hannover

Carl-Neuberg-Str. 1, 30625 Hannover, Deutschland

david.sascha@mh-hannover.de

\section{Einhaltung ethischer Richtlinien}

Interessenkonflikt. S. David und F.M. Brunkhorst geben an, dass kein Interessenkonflikt besteht.

Dieser Beitrag beinhaltet keine von den Autoren durchgeführten Studien an Menschen oder Tieren.

\section{Literatur}

1. ARISE Investigators, ANZICS Clinical Trials Group, Peake SL et al (2014) Goal-directed resuscitation for patients with early septic shock. N Engl J Med 371:1496-1506. https://doi.org/10.1056/ NEJMoa 1404380

2. Bone RC, Balk RA, Cerra FB et al (1992) Definitions for sepsis and organ failure and guidelines for the use of innovative therapies in sepsis. The ACCP/SCCM Consensus Conference Committee. American College of Chest Physicians/Society of Critical Care Medicine. Chest 101(6):1644-1655

3. Cherpanath TGV, Hirsch A, Geerts BF et al (2016) Predicting fluid responsiveness by passive leg raising: a systematic review and meta-analysis of 23 clinical trials. Crit Care Med 44:981-991. https:// doi.org/10.1097/CCM.0000000000001556

4. Levy MM, Fink MP, Marshall JC et al (2003) 2001 SCCM/ESICM/ACCP/ATS/SIS International Sepsis Definitions Conference. Intensive Care Med 29:530-538. https://doi.org/10.1007/s00134003-1662-x

5. Mouncey PR, Osborn TM, Power GS et al (2015) Trial of early, goal-directed resuscitation for septic shock. N Engl J Med 372:1301-1311. https://doi. org/10.1056/NEJMoa1500896

6. Perner A, Haase N, Guttormsen AB et al (2012) Hydroxyethyl starch 130/0.42 versus Ringer's acetate in severe sepsis. N Engl J Med 367:124-134. https://doi.org/10.1056/NEJMoa1204242

7. Investigators PCES, Yealy DM, Kellum JA et al (2014) A randomized trial of protocol-based care for early septic shock. N Engl J Med 370:1683-1693. https:// doi.org/10.1056/NEJMoa1401602

8. Rhodes A, Evans LE, Alhazzani W et al (2017) Surviving sepsis campaign: international guidelines for management of sepsis and septic shock: 2016 Intensive Care Med 43(3):304-377. https://doi. org/10.1007/s00134-017-4683-6

9. Rhodes A, Evans LE, Alhazzani W et al (2017) Surviving sepsis campaign: international guidelines for management of sepsis and septic shock: 2016. Crit Care Med 45:486-552. https://doi.org/10.1097/ CCM. 0000000000002255

10. Rivers E, Nguyen B, Havstad Setal (2001) Earlygoaldirected therapy in the treatment of severe sepsis and septic shock. N Engl J Med 345:1368-1377. https://doi.org/10.1056/NEJMoa010307

11. Seymour CW, Gesten F, Prescott HC et al (2017) Time to treatment and mortality during mandated emergency care for sepsis. N Engl J Med 376:2235-2244. https://doi.org/10.1056/ NEJMoa1703058

12. Seymour CW, Liu VX, Iwashyna TJ et al (2016) Assessment of clinical criteria for sepsis: for the third international consensus definitions for sepsis and septic shock (sepsis-3). JAMA 315:762-774. https://doi.org/10.1001/jama.2016.0288

13. Shankar-Hari M, Phillips GS, Levy ML et al (2016) Developing a new definition and assessing new clinical criteria for septic shock: for the third international consensus definitions for sepsis and septic shock (sepsis-3). JAMA 315(8):775-787. https://doi.org/10.1001/jama.2016.0289

14. Singer M, Deutschman CS, Seymour CWetal (2016) The third international consensus definitions for sepsis and septic shock (sepsis-3). JAMA 315(8):801-810
Hier steht eine Anzeige. Springer 